\title{
Optimization of annealing to decrease quantity of radiation defects in a bipolar transistor
}

\author{
E. L. Pankratov
}

Received: 21 May 2011 / Accepted: 22 July 2011/Published online: 30 August 2011

(C) The Author(s) 2011. This article is published with open access at Springerlink.com

\begin{abstract}
It has recently been shown that manufacturing of diffusive- and implanted-junction bipolar transistors in semiconductor heterostructure and optimization of annealing give us possibility to increase compactness of dopant distributions. In this paper, we analyze the possibility of decreasing the quantity of radiation defects by choosing regimes of annealing.
\end{abstract}

Keywords Implanted-junction rectifiers . Optimization of manufactoring of rectifiers . Decreasing of quantity of radiation defects

\section{Introduction}

One of the intensive solving problems of solid-state electronics is decreasing the dimensions of elements of integrated circuits (IC) (Gusev and Gusev 1991; Grebene 1983; Lachin and Savelov 2001; Gotra 1991). Another intensive solving problem is increasing the performance of such elements of IC as $p-n$-junctions and their systems (bipolar transistors and thyristors).

To fabricate $p-n$-junctions and their systems, some approaches can be used. One of the approaches is implantation of ions of dopant in a semiconductor sample or epitaxial layer (EL) of a heterostructure $(\mathrm{H})$. It has been recently shown (see Pankratov 2008a, b) that implantation of dopant in a two-layer (TwL) $\mathrm{H}$ or in a three-layer (ThL) $\mathrm{H}$ at appropriately chosen energy of ions, thickness and materials of layers of $\mathrm{H}$ and optimal value of annealing time gives us

E. L. Pankratov $(\square)$

Nizhny Novgorod State University of Architecture

and Civil Engineering, 65 Il'insky street,

Nizhny Novgorod 603950, Russia

e-mail: elp2004@mail.ru the possibility to increase homogeneity of dopant distribution in doped area and sharpness of the $p-n$-junctions. Increasing the homogeneity gives us the possibility to decrease local overheats in the doped area during operation of the $p-n$ junction or to decrease the depth of the $p-n$-junction at fixed tolerance on the value of the local overheats. A disadvantage of ion implantation is radiation damage of $\mathrm{H}$. The main aim of the present paper is determination of the conditions to decrease the radiation damages or to decrease the influence of the damages on the characteristics of devices.

\section{Statement of the problem}

In this paper we consider a ThLH, which consists of a substrate (S) and two epitaxial layers $\mathrm{EL}_{1}$ and $\mathrm{EL}_{2}$ (see Fig. 1). The type of conductivity of $S$ and other parameters (thickness, dopant diffusion coefficient, etc.) are known. Thicknesses and other parameters of $\mathrm{EL}_{1}$ and $\mathrm{EL}_{2}$ are known. A dopant has been implanted in the $\mathrm{EL}_{1}$ to produce the type of conductivity, which is reverse to the type of conductivity of S. Another dopant has been also implanted in $\mathrm{EL}_{2}$ to produce the type of conductivity, which coincides with the type of conductivity of S. Further annealing of radiation defects was done. After appropriate choosing of the thicknesses of the epitaxial layers and energies of ions, the dopants achieve the interfaces between $\mathrm{EL}_{1}$ and $\mathrm{S}$ and also $\mathrm{EL}_{1}$ and $\mathrm{EL}_{2}$. If the interfaces were not achieved by the dopants, additional annealing to shift the $p-n$-junctions to the interfaces attracted interest. If the dopants achieved the interface, maximal compromise between increasing the homogeneity of dopant distribution and sharpness of the $p-n$-junctions was obtained. Let us consider an alternative approach to annealing. In the framework of the alternative approach, the first dopant was implanted in $\mathrm{EL}_{1}$. Further 


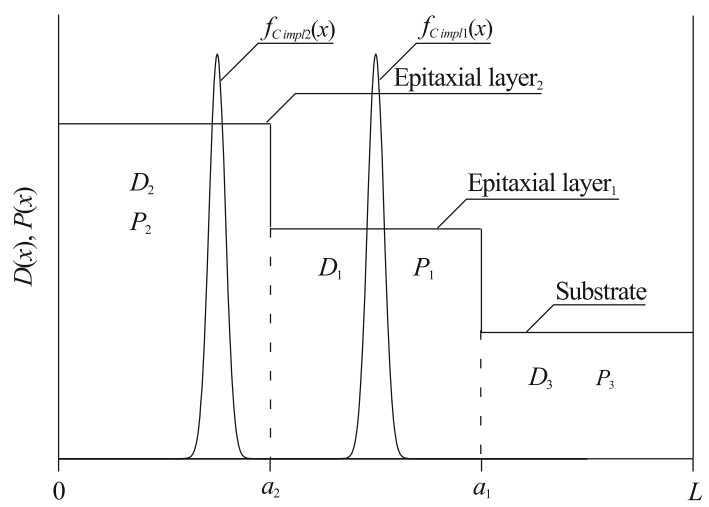

Fig. 1 Heterostructure, which consist of a substrate and two epitaxial layers

radiation defects were annealed. After that, another dopant was implanted in $\mathrm{EL}_{2}$ and new defects were annealed. The main aim of the present paper is comparison of restoring abilities of ThLH in the situations.

$$
\begin{aligned}
D_{C k}= & D_{L k}(x, T) \prod_{j=1}^{k}\left[1+\xi_{j} \frac{C_{j}^{\gamma_{j}}(x, t)}{P_{j}^{\gamma_{j}}(x, T)}\right] \\
& \times\left[1+\zeta_{1} \frac{V(x, t)}{V^{*}}+\zeta_{2} \frac{V^{2}(x, t)}{\left(V^{*}\right)^{2}}\right] .
\end{aligned}
$$

Here, $P_{k}(x, T)$ is the limit of solubility of the $k$ th type dopant in $\mathrm{H} ; D_{L k}(x, T)$ is the diffusion coefficient of the $k$ th type dopant for low level of doping; parameter $\gamma_{k}$, which depends on the properties of materials of $\mathrm{H}$, can be the integer usually in the interval $\gamma_{k} \in[1,3]$ (Gotra 1991); $T$ is the temperature of annealing; $V(x, t)$ is the spatiotemporal distribution of concentration of vacancies; $V^{*}$ is the equilibrium distribution of concentration of vacancies. Spatiotemporal distributions of concentration of defects were determined by the following system of equations (Zorin et al. 1975, Fahey et al. 1989)

with boundary and initial conditions

$$
\left\{\begin{aligned}
\frac{\partial I(x, t)}{\partial t}= & \frac{\partial}{\partial x}\left[D_{I}(x, T) \frac{\partial I(x, t)}{\partial x}\right]-k_{I, V}(x, T) I(x, t) V(x, t)-k_{I, I}(x, T) I^{2}(x, t) \\
& =-\frac{\partial J_{I}(x, t)}{\partial x}-k_{I, V}(x, T) I(x, t) V(x, t)-k_{I, I}(x, T) I^{2}(x, t) \\
\frac{\partial V(x, t)}{\partial t}= & \frac{\partial}{\partial x}\left[D_{V}(x, T) \frac{\partial V(x, t)}{\partial x}\right]-k_{I, V}(x, T) I(x, t) V(x, t)-k_{V, V}(x, T) V^{2}(x, t) \\
& =-\frac{\partial J_{V}(x, t)}{\partial x}-k_{I, V}(x, T) I(x, t) V(x, t)-k_{V, V}(x, T) V^{2}(x, t)
\end{aligned}\right.
$$

\section{Method of solution}

Let us determine the spatiotemporal distribution of dopant concentration to solve our aim. We determine the distribution by solving the second Fick's law (Gusev and Gusev 1991; Grebene 1983; Lachin and Savelov 2001; Gotra 1991)

$\frac{\partial C_{k}(x, t)}{\partial t}=\frac{\partial}{\partial x}\left[D_{C k} \frac{\partial C_{k}(x, t)}{\partial x}\right]$

with boundary and initial conditions

$J_{C k}(0, t)=0, \quad J_{C k}(L, t)=0, \quad C_{k}(x, 0)=f_{C k}(x)$.

Here, $C_{k}(x, t)$ is the spatiotemporal distribution of the $k$ th type dopant concentration; $J_{C k}(x, t)$ is the spatiotemporal distribution of the $k$ th type dopant flow; $D_{C k}$ is the dopant diffusion coefficient. The value of dopant diffusion coefficient depends on the properties of materials of layers in $\mathrm{H}$, on the rate of heating and cooling of $\mathrm{SH}$ and on spatiotemporal distributions of dopant and defect concentrations. The concentration dependence of the diffusion coefficient could be approximated by the following functions (Gotra 1991; Zorin et al. 1975)
$J_{I}(0, t)=0, \quad J_{I}(L, t)=0, \quad I(x, 0)=f_{I}(x)$,

$J_{V}(0, t)=0, \quad J_{V}(L, t)=0, \quad V(x, 0)=f_{V}(x)$.

Here $I(x, t)$ is the spatiotemporal distribution of concentration of interstitials; $D_{I}(x, T)$ and $D_{V}(x, T)$ are diffusion coefficients of vacancies and interstitials; quadratic terms in Eq. 4 correspond to generation of divacancies and diinterstitials (see, for example Pankratov 2008c and appropriate references in the paper); $k_{I, V}(x, T)$ is the recombination parameter; $k_{I, I}(x, T)$ and $k_{V, V}(x, T)$ are parameters of generation of diinterstitials and divacancies, respectively. In this paper, in comparison with Pankratov (2008a, b), generation complexes of radiation defects have been accounted for.

Let us transform Eqs. 1-4 to the following integral form

$$
\begin{aligned}
& C_{k}(x, t)=C_{k}(x, t)+\frac{1}{L}\left\{F_{C k}+\int_{0}^{t} D_{L k}(x, T) W(x, \tau)\right. \\
& \left.\quad \times \prod_{j=1}^{k}\left[1+\xi_{k} \frac{C_{k}^{\gamma_{k}}(x, \tau)}{P_{k}^{\gamma_{k}}(x, T)}\right] \frac{\partial C_{k}(x, \tau)}{\partial x} \mathrm{~d} \tau-\int_{L}^{x} C_{k}(v, t) \mathrm{d} v\right\},
\end{aligned}
$$




$$
\left\{\begin{aligned}
I(x, t)= & I(x, t)+\frac{1}{L}\left\{\int_{0}^{t} D_{I}(x, T) \frac{\partial I(x, \tau)}{\partial x} \mathrm{~d} \tau-\int_{0}^{t} k_{I, V}(v, T) I(v, \tau) V(v, \tau) \mathrm{d} v \mathrm{~d} \tau+F_{I}(x)\right. \\
& -\int_{L}^{x} I(v, t) \mathrm{d} v-\int_{0}^{t} k_{I, I}(v, T) I^{2}(v, \tau) \mathrm{d} v \mathrm{~d} \tau \\
V(x, t)= & V(x, t)+\frac{1}{L}\left\{\int_{0}^{t} D_{V}(x, T) \frac{\partial V(x, \tau)}{\partial x} \mathrm{~d} \tau-\int_{0}^{t} k_{I, V}(v, T) I(v, \tau) V(v, \tau) \mathrm{d} v \mathrm{~d} \tau+F_{V}(x)\right. \\
& -\int_{L}^{x} V(v, t) \mathrm{d} v-\int_{0}^{t} k_{V, V}(v, T) I^{2}(v, \tau) \mathrm{d} v \mathrm{~d} \tau .
\end{aligned}\right.
$$

Here, $W(x, t)=\left[1+\zeta_{1} \frac{V(x, t)}{V^{*}}+\zeta_{2} \frac{V^{2}(x, t)}{\left(V^{*}\right)^{2}}\right] ; F_{\rho}=\int_{L}^{x} f_{\rho}(v) \mathrm{d} v$; $\rho=C, I, V$.

Let us determine the solution of Eqs. 6 and 7 using the method of averaging of function corrections (Sokolov 1955) with a decreased number of iteration steps (Pankratov 2005). In the framework of the approach, we consider the solutions of Eqs. 1 and 4 with averaged value $D_{0 \mu}$ of diffusion coefficients $D_{\mu}(x, T)(\mu=L, I, V)$ and zero value of parameter $\xi_{k}, \zeta_{j}, K_{I, V}(x, T), K_{I, I}(x, T), K_{V, V}(x, T)$ instead of the averaged value of concentrations of dopants and radiation defects $\alpha_{1 C}, \alpha_{1 I}$ and $\alpha_{1 V}$ as a more exact initial-order approximation of solution of Eqs. 6 and 7. The solutions of Eqs. 1 and 4 could be written in the form $\widetilde{\rho}(x, t)=\frac{1}{L}\left[F_{0 \rho}+2 \sum_{n=1}^{\infty} F_{n \rho} c_{n}(x) e_{n \rho}(t)\right]$.

Here, $\quad c_{n}(x)=\cos \left(\frac{\pi n x}{L}\right) ; F_{n \rho}=\int_{0}^{L} f(x) c_{n}(x) \mathrm{d} x ; e_{n \rho}(t)=$ $\exp \left(-\frac{\pi^{2} n^{2} D_{n \mu} t}{L^{2}}\right)$ (index $L$ of the function $e_{n \rho}(t)$ has been replaced on $C$ ). The first-order approximations of dopants and radiation defects concentrations could be obtained by substitution of the relation in Eq. 7 into the right side of Eqs. 6 and 7 instead of the functions $\rho(x, t)$. After the substitution, the first-order approximations take the form

$$
\left\{\begin{aligned}
I_{1}(x, t)= & \frac{1}{L}\left[F_{0 I}+2 \sum_{n=1}^{\infty} F_{n I} c_{n}(x) e_{n I}(t)\right]+\frac{1}{L}\left\{-\frac{2 \pi}{L^{2}} \sum_{n=1}^{\infty} n F_{n I} s_{n}(x) \int_{0}^{t} D_{I}(x, T) e_{n I}(\tau) \mathrm{d} \tau\right. \\
& -\frac{1}{L^{2}} \int_{0}^{t} \int_{L}^{x} k_{I_{V}}(v, T)\left[F_{0 I}+2 \sum_{n=1}^{\infty} F_{n I} c_{n}(v) e_{n I}(\tau)\right]\left[F_{0 V}+2 \sum_{n=1}^{\infty} F_{n V} c_{n}(v) e_{n V}(\tau)\right] \mathrm{d} v \mathrm{~d} \tau+F_{I}(x) \\
& -\frac{1}{L^{2}} \int_{0}^{t} \int_{L}^{x} k_{I_{I}}(v, T)\left[F_{0 I}+2 \sum_{n=1}^{\infty} F_{n I} c_{n}(v) e_{n I}(\tau)\right]^{2} \mathrm{~d} v \mathrm{~d} \tau+\left[F_{0 I}-F_{0 I} \frac{x}{L}+\frac{2}{\pi} \sum_{n=1}^{\infty} \frac{F_{n I}}{n} s_{n}(x) e_{n I}(t)\right] \\
V_{1}(x, t)= & \frac{1}{L}\left[F_{0 V}+2 \sum_{n=1}^{\infty} F_{n V} c_{n}(x) e_{n V}(t)\right]+\frac{1}{L}\left\{-\frac{2 \pi}{L^{2}} \sum_{n=1}^{\infty} n F_{n V} s_{n}(x) \int_{0}^{t} D_{V}(x, T) e_{n V}(\tau) \mathrm{d} \tau\right. \\
& -\frac{1}{L^{2}} \int_{0}^{t} \int_{L}^{x} k_{I_{V}}(v, T)\left[F_{0 I}+2 \sum_{n=1}^{\infty} F_{n I} c_{n}(v) e_{n I}(\tau)\right]\left[F_{0 V}+2 \sum_{n=1}^{\infty} F_{n V} c_{n}(v) e_{n V}(\tau)\right] \mathrm{d} v \mathrm{~d} \tau+F_{V}(x) \\
& -\frac{1}{L^{2}} \int_{0}^{t} \int_{L}^{x} k_{V_{V}}(v, T)\left[F_{0 V}+2 \sum_{n=1}^{\infty} F_{n V} c_{n}(v) e_{n V}(\tau)\right] \mathrm{d} v \mathrm{~d} \tau+\left[F_{0 V}-F_{0 V} \frac{x}{L}+\frac{2}{\pi} \sum_{n=1}^{\infty} \frac{F_{n V}}{n} s_{n}(x) e_{n V}(t)\right],
\end{aligned}\right.
$$




$$
\begin{aligned}
& C_{1 k}(x, t)=\frac{1}{L}\left[F_{0 C_{k}}+2 \sum_{n=1}^{\infty} F_{n C_{k}} c_{n}(x) e_{n C_{k}}(t)\right] \\
& +\frac{1}{L}\left(F_{C}(x)+F_{0 C_{k}}\left(1-\frac{x}{L}\right)-\frac{2}{\pi} \sum_{n=1}^{\infty} e_{n C k}(t) \frac{F_{n C_{k}}}{n}\right. \\
& \times s_{n}(x)-2 \pi \frac{1}{L^{2}} \int_{0}^{t} W(x, \tau) D_{L k}(x, T) \\
& \times \prod_{j=1}^{k}\left\{1+\frac{\xi_{j}^{\gamma_{j}}}{L^{\gamma_{j}} P_{j}^{\gamma_{j}}(x, T)}\left[F_{0 C k}+2 \sum_{n=1}^{\infty} F_{n C k} c_{n}(x) e_{n C k}(\tau)\right]^{\gamma_{k}}\right\} \\
& \left.\times \sum_{n=1}^{\infty} n F_{n C} s_{n}(x) e_{n C}(\tau) \mathrm{d} \tau\right) .
\end{aligned}
$$

Let us to determine the approximations of dopants and radiation defects concentrations with the higher order by using the standard procedure of the method of averaging of function corrections (Pankratov 2008c; Sokolov 1955), i.e., by substitution of the functions $\rho(x, t)$ in the right side of Eqs. 6 and 7 in the following sum $\alpha_{i \rho}+\rho_{i-1}(x, t)$, where $i$ is the order of approximation. The substitution gives us the possibility of obtaining the second-order approximations of concentrations in the following form

$$
\begin{aligned}
C_{2 k}(x, t)= & \alpha_{2 C k}+C_{1 k}(x, t)+\frac{1}{L}\left(F_{C k}(x)+\int_{0}^{t} D_{L k}(x, T)\right. \\
& \times \prod_{j=1}^{k}\left\{1+\xi_{j} \frac{\left[\alpha_{2 C j}+C_{1 j}(x, \tau)\right]^{\gamma_{j}}}{P_{j}^{\gamma_{j}}(x, T)}\right\} \\
& \left.\times \frac{\partial C_{1 k}(x, \tau)}{\partial x} \mathrm{~d} \tau-\alpha_{2 C k}(x-L)-\int_{L}^{x} C_{1 k}(v, t) \mathrm{d} v\right),
\end{aligned}
$$

The parameter $\alpha_{2 \rho}$ could be calculated by the standard relation (Pankratov 2008c; Sokolov 1955)

$\alpha_{2 \rho}=\frac{1}{L \Theta} \int_{0}^{\Theta} \int_{0}^{L}\left[\rho_{i}(x, t)-\rho_{i-2}(x, t)\right] \mathrm{d} x \mathrm{~d} t$.

One can obtain the following equation to determine the parameter $\alpha_{2 C}$ for a fixed value of parameter $\gamma_{k}$ by substituting Eqs. 10 and 11 in the relation in Eq. 13

$$
\begin{aligned}
& \int_{0}^{\Theta}(\Theta-t) \int_{0}^{L} D_{L k}(x, T) W(x, t) \\
& \times \prod_{j=1}^{k}\left\{1+\xi_{j} \frac{\left[\alpha_{2 C j}+C_{1 j}(x, t)\right]^{\gamma_{j}}}{P_{j}^{\gamma_{j}}(x, T)}\right\} \frac{\partial C_{1 k}(x, \tau)}{\partial x} \mathrm{~d} x \mathrm{~d} t \\
& +\alpha_{2 C k} \Theta \frac{L^{2}}{2}+\int_{0}^{\Theta} \int_{0}^{L} x C_{1}(x, t) \mathrm{d} x \mathrm{~d} t-\Theta \int_{0}^{L} x C_{1 k}(x, t) \mathrm{d} x=0 .
\end{aligned}
$$

Values of the parameter $\alpha_{2 C k}$ for several values of parameter $\gamma_{k}$ are presented in the Appendix. By substitution of Eqs. 9 and 12 in the relation in Eq. 13, one can obtain the relations for parameters $\alpha_{2 I}$ and $\alpha_{2 V}$ in the following form

$$
\begin{aligned}
\alpha_{2 I}= & \frac{1}{2} \sqrt{\frac{\left(a_{2}-A a_{1}\right)^{2}}{4 a_{1}^{2}}-4\left(y+\frac{a_{4}-a_{2} y}{A a_{1}}\right)}+\frac{A a_{1}-a_{2}}{4 a_{1}}, \\
\alpha_{2 V}= & -\left[\alpha_{2 I}^{2} S_{I, I 0100}+\alpha_{2 I}\left(S_{I, V 1101}+2 S_{I, I 0110}+\Theta \frac{L^{2}}{2}\right)\right. \\
& +S_{I, V 1111}+S_{I, I 0120}+N_{I}+Q_{I} \\
& \left.+\Theta \int_{0}^{L} F_{I}(x) d x\right]\left(\alpha_{2 I} S_{I, V 1100}+S_{I, V 1110}\right)^{-1} .
\end{aligned}
$$

$$
\left\{\begin{aligned}
I_{2}(x, t)= & \alpha_{2 I}+I_{1}(x, t)+\frac{1}{L}\left(F_{I}+\int_{0}^{t} D_{I}(x, T) \frac{\partial I_{1}(x, \tau)}{\partial x} \mathrm{~d} \tau-\int_{0}^{t} \int_{L}^{x}\left[\alpha_{2 I}+I_{1}(v, \tau)\right]\left[\alpha_{2 V}+V_{1}(v, \tau)\right]\right. \\
& \left.\times k_{I, V}(v, T) \mathrm{d} v \mathrm{~d} \tau-\int_{0}^{t} \int_{L}^{x} k_{I, I}(v, T)\left[\alpha_{2 I}+I_{1}(v, \tau)\right]^{2} \mathrm{~d} v \mathrm{~d} \tau-\alpha_{2 I}(x-L)-\int_{0}^{t} \int_{L}^{x} I_{1}(v, t) \mathrm{d} v\right) \\
V_{2}(x, t)= & \alpha_{2 V}+V_{1}(x, t)+\frac{1}{L}\left(\int_{0}^{t} D_{V}(x, T) \frac{\partial V_{1}(x, \tau)}{\partial x} \mathrm{~d} \tau-\int_{0}^{t} \int_{L}^{x}\left[\alpha_{2 I}+I_{1}(v, \tau)\right]\left[\alpha_{2 V}+V_{1}(v, \tau)\right]\right. \\
& \left.\times k_{I, V}(v, T) \mathrm{d} v \mathrm{~d} \tau-\int_{0}^{t} \int_{L}^{x} k_{V, V}(v, T)\left[\alpha_{2 V}+V_{1}(v, \tau)\right]^{2} \mathrm{~d} v \mathrm{~d} \tau-\alpha_{2 V}(x-L)-\int_{0}^{t} \int_{L}^{x} V_{1}(v, t) d v+F_{V}\right) .
\end{aligned}\right.
$$


Here, $S_{\phi, \varphi, i j k l}=\int_{0}^{\Theta}(\Theta-t) \int_{0}^{L} x^{i}\left[k_{\phi, \varphi}(x, T)\right]^{j} I_{1}^{k}(x, t) V_{1}^{l}(x, t)$ $\mathrm{d} x \mathrm{~d} t, Q_{\rho}=\int_{0}^{\Theta}(\Theta-t) \int_{0}^{L} D_{\rho}(x, T) \quad \frac{\partial \rho_{1}(x, t)}{\partial x} \mathrm{~d} x \mathrm{~d} t, \quad N_{\rho}=$ $\int_{0}^{\Theta} \int_{0}^{L} x \rho_{1}(x, t) \mathrm{d} x \mathrm{~d} t, a_{1}=S_{I, I 0100}\left(S_{V, V 0100} S_{I, I 0100}-S_{I, V 1100}^{2}\right)$, $a_{2}=\left[\left(2 S_{I, I 0110}+S_{I, V 1101}+\Theta \frac{L^{2}}{2}\right) \times 2 S_{V, V 0100} S_{I, I 0100}-S_{I, I 0100}\right.$ $\left(S_{I, V 1110}+2 S_{V, V 1101}+S_{I, V 1100} S_{I, V 1110}+\Theta \frac{L^{2}}{2}\right)+S_{I, V 1101}$ $S_{I, V 1100}^{2}-\left(\frac{L^{2}}{2} \times \Theta+S_{I, V 1110}+2 S_{V, V 1101}+S_{I, V 1100} S_{I, V 1110}\right)$ $\left.S_{I, V 1100}^{2}\right], a_{3}=\left\{S_{I, V 1100}^{2}\left[S_{I, V 1111}+S_{I, V 1001}+S_{I, V 0102}+Q_{V}+\right.\right.$ $\left.\int_{0}^{L} F_{I}(x) \mathrm{d} x\right]-\left(S_{I, V 1110}+\Theta \frac{L^{2}}{2}+2 S_{V, V 1101}+S_{I, V 1100} S_{I, V 1110}\right)^{2}$ $-S_{I, V 1100}^{2}\left[S_{I, I 0120}+Q_{I}+N_{I}+S_{I, V 1111}+\int_{0}^{L} F_{I}(x) \mathrm{d} x\right]+$ $2 S_{I, V 1101} S_{I, V 1100} S_{I, V 1110}-S_{I, V 1110} S_{I, I 0100}\left(S_{I, V 1110}+2 S_{V, V 1101}\right.$ $\left.+\Theta \frac{L^{2}}{2}\right)+S_{V, V 0100}\left(S_{I, I 0110}+S_{I, V 1101}+\Theta \frac{L^{2}}{2}\right)+2 S_{I, I 0100}$ $\left.S_{V, V 0100}\left[\Theta \int_{0}^{L} F_{I}(x) \mathrm{d} x+Q_{I}+S_{I, I 0120}+S_{I, V 1111}+N_{I}\right]^{2}\right\}, a_{4}=$ $S_{I, V 1101} S_{I, V 1110}^{2}+S_{I, V 1100}\left[\int_{0}^{L} F_{I}(x) \mathrm{d} x+S_{I, V 1111}+S_{I, V 1100}+\right.$ $\left.S_{I, V 1001}+S_{I, V 0102}+Q_{V}\right] \times 2 S_{I, V 1110}-S_{I, V 1110}\left(S_{I, V 1110}+\right.$ $\left.2 S_{V, V 1101}+\Theta \frac{L^{2}}{2}\right)\left(S_{I, V 1110}+S_{V, V 1101}+S_{I, V 1100} S_{I, V 1110}+\right.$ $\left.\Theta \frac{L^{2}}{2}\right)-\left\{\Theta \int_{0}^{L} F_{I}(x) \mathrm{d} x+S_{I, I 0120}+S_{I, V 1111}+S_{V, V 0100}[\right.$ $\left.\left.\Theta \int_{0}^{L} F_{I}(x) \mathrm{d} x+S_{I, I 0120}+S_{I, V 1111}+Q_{I}+N_{I}\right]+N_{I}+Q_{I}\right\}$ $\left(S_{I, V 1100} S_{I, V 1110}+\Theta \frac{L^{2}}{2}+2 S_{V, V 1101}+S_{I, V 1110}\right)+2 S_{V, V 0100}[$ $\left.\Theta \int_{0}^{L} F_{I}(x) \mathrm{d} x+S_{I, V 0120}+N_{I}+Q_{I}+S_{I, V 1121}\right]\left(S_{I, V 0110}+\right.$ $\left.S_{I, V 1101}+\Theta \frac{L^{2}}{2}\right), a_{5}=S_{I, V 1110}^{2}\left[\int_{0}^{L} F_{I}(x) \mathrm{d} x+S_{I, V 1111} S_{I, V 1001}+\right.$ $\left.S_{I, V 0102}+Q_{V}\right]-S_{I, V 1110}\left(S_{I, V 1110}+S_{V, V 1101}+\Theta \frac{L^{2}}{2}\right)\left(S_{I, I 0120}+\right.$ $\left.S_{I, V 1111}+\Theta \int_{0}^{L} F_{I}(x) \mathrm{d} x+Q_{I}+N_{I}\right)+S_{V, V 010}\left[N_{I}+\Theta \int_{0}^{L}\right.$ $\left.F_{I}(x) \mathrm{d} x+Q_{I}+S_{I, I 0120}+S_{I, V 1111}\right]^{2}, y=\sqrt[3]{\sqrt{\widetilde{q}^{2}+\widetilde{p}^{3}}-\widetilde{q}}-$ $\sqrt[3]{\sqrt{\widetilde{q}^{2}+\widetilde{p}^{3}}+\widetilde{q}}+\frac{a_{3}}{6 a_{1}}, \widetilde{p}=\left(\frac{a_{2} a_{4}}{4 a_{1}^{2}}-\frac{a_{5}}{a_{1}}\right) \frac{1}{3}-\frac{a_{2}^{2}}{36 a_{1}^{2}}$, $\widetilde{q}=\frac{a_{3}}{6 a_{1}^{2}}\left(\frac{a_{2} a_{4}}{4 a_{1}^{2}}-\frac{a_{5}}{a_{1}}\right)+\frac{a_{5}}{8 a_{1}}\left(4 \frac{a_{2}}{a_{1}}-\frac{a_{2}^{2}}{a_{1}^{2}}\right)-\frac{a_{4}^{2}}{8 a_{1}^{2}}-\frac{a_{3}^{3}}{216 a_{1}^{3}}, \quad A=$ $\sqrt{8 y+\frac{a_{2}^{2}}{a_{1}^{2}}-4 \frac{a_{3}}{a_{1}}}$.

Let us analyze the redistribution of dopants in $\mathrm{H}$. The obtained analytical results usually gives us the possibility to analyze demonstrably the redistribution of dopants during annealing of radiation defects as a functions of parameters. The second advantage of analytical solution is decreasing the calculation time of dopant and defect distribution, because calculation of the sums of elementary functions needs lesser time than numerical solution of differential or integral equation. On the other hand, using numerical approaches gives us the possibility to obtain more exact results. Therefore, we used both analytical and numerical approaches to analyze redistributions of dopants (we used numerical solution to check the analytical solution).

\section{Discussion}

Let us analyze the redistribution of dopant in the $\mathrm{H}$ (see Fig. 1). During the analysis, it is worthwhile to take into account matching energy of ions and thicknesses of epitaxial layers to achieve or almost achieve the appropriate interfaces of $\mathrm{H}$ by dopants during annealing of radiation defects. In this situation, the first dopant should achieve the interface between $\mathrm{EL}_{1}$ and $\mathrm{S}$ and also between $\mathrm{EL}_{1}$ and

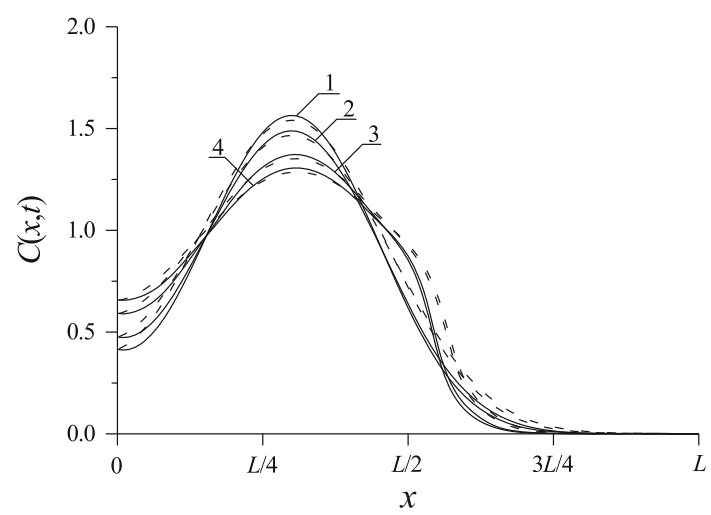

Fig. 2 Distribution of dopant in two-layer heterostructure. Curve 1 is the distribution of dopant in the homogenous sample. Curves 2-4 are distributions of dopant in two-layer heterostructure for the case, when diffusion coefficient of dopant in the epitaxial layer is larger than the diffusion coefficient of dopant in the substrate. Increasing the number of curves corresponds to increasing the difference between diffusion coefficients. In this case, the position of the layer interface is $a=L / 2$

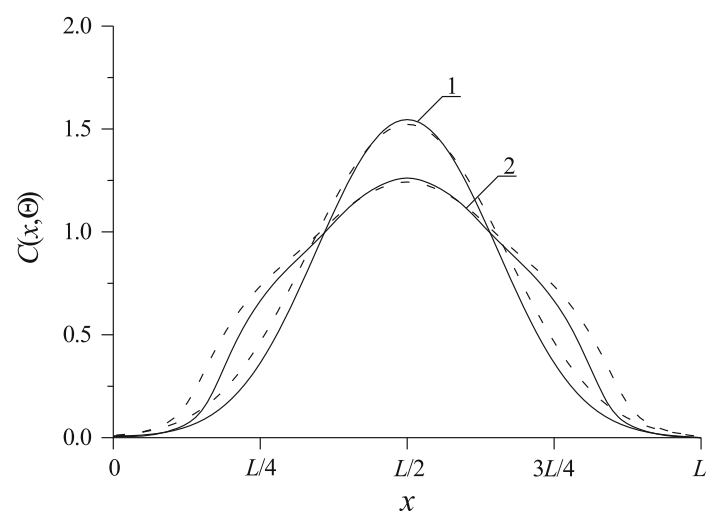

Fig. 3 Distribution of dopant in the three-layer heterostructure. Curve 1 is distribution of dopant in the homogenous sample. Curve 2 is the distribution of the dopant in the three-layer heterostructure for the case, when diffusion coefficient of the dopant in the epitaxial layer is larger than the diffusion coefficient of the dopant in the substrate. In this case, the positions of the layer interfaces are $a_{1}=3 L / 4$ and $a_{2}=L / 4$ 
$\mathrm{EL}_{2}$; the second dopant should achieve the interface between $\mathrm{EL}_{1}$ and $\mathrm{EL}_{2}$. If the dopants almost achieved the interfaces, additional annealing of dopants attracted interest to the shift of the $p-n$-junctions to the appropriate interfaces. The achievement attracted an interest to

\section{Appendix}

Values of parameters $\alpha_{2 c k}$ for different magnitudes of parameter $\gamma_{k}: \gamma_{k}=1$

$\alpha_{2 c k}=\frac{\Theta \int_{0}^{L} x f_{C k}(x) \mathrm{d} x-\int_{0}^{\Theta}(\Theta-t) \int_{0}^{L} R_{00 k}(x, t) \mathrm{d} x \mathrm{~d} t+\int_{0}^{\Theta} \int_{0}^{L} C_{1 k}(x, t) \mathrm{d} x \mathrm{~d} t-\xi_{k} \int_{0}^{\Theta}(\Theta-t) \int_{0}^{L} R_{11 k}(x, t) \mathrm{d} x \mathrm{~d} t}{\xi_{k} \int_{0}^{\Theta}(\Theta-t) \int_{0}^{L} R_{01 k}(x, t) \mathrm{d} x \mathrm{~d} t+\Theta L^{2} / 2}$,

increase the sharpness of the $p-n$-junctions and at one time to increase the homogeneity of dopant distributions in the doped area (see, for example, Figs. 2, 3 and (Pankratov 2008a, b). Both the effects (increasing the sharpness of $p-n$-junction, and increasing the homogeneity of dopant concentrations) will be obtained in both situations: after annealing of all radiation defects, after implantation of both dopants and after annealing of individual groups of radiation defects, particularly after each ion implantation. However, annealing of each individual groups of defects gives us the possibility to accelerate recrystallization of $\mathrm{H}$ in the area of collision of both distributions of radiation defects. At one time during this type of annealing, one can obtain decreasing generation of complexes of defects.

\section{Conclusion}

In this paper we compare two types of annealing of radiation defects, which have been generated in a semiconductor heterostructure during ion implantation to produce a bipolar transistor. The first of them is one-stage implantation (implantation of all dopant into the heterostructure). The second of them is two-stage implantation (at the first stage only one half of the dopant is implanted, then radiation defects are annealed; at the second stage, the second half of the dopant is implanted, then radiation defects are annealed again). The comparison gives us the possibility to determine that the second type of implantation corresponds to larger decrease in radiation damage.

Acknowledgments This work has been supported by a grant from the Nizhny Novgorod State University of Architecture and Civil Engineering (project 241) and a grant from the President of Russia (project MK-548.2010.2).

Open Access This article is distributed under the terms of the Creative Commons Attribution License which permits any use, distribution and reproduction in any medium, provided the original author(s) and source are credited. where $R_{i j m}(x, t)=D_{L m}(x, T) W(x, t) \frac{C_{1 m}^{j}(x, t)}{P_{k}^{j}(x, T)} \frac{\partial C_{1 m}(x, t)}{\partial x}, \gamma_{k}=2$

$\alpha_{2 c k}=\frac{1}{2}\left(\sqrt{a_{7}^{2}-4 a_{6} a_{8}}-a_{7}\right)$

where $\quad a_{6}=\xi_{k} \int_{0}^{\Theta}(\Theta-t) \int_{0}^{L} R_{02 k}(x, t) \mathrm{d} x \mathrm{~d} t, a_{7}=2 \xi_{k} \int_{0}^{\Theta}$ $(\Theta-t) \quad \int_{0}^{L} R_{12 k}(x, t) \mathrm{d} x \mathrm{~d} t, a_{8}=\int_{0}^{\Theta} \quad \int_{0}^{L} R_{00 k}(x, t) \mathrm{d} x \times$ $(\Theta-t) \mathrm{d} t+\xi_{k} \int_{0}^{\Theta}(\Theta-t) \quad \int_{0}^{L} R_{22 k}(x, t) \mathrm{d} x \mathrm{~d} t+\int_{0}^{\Theta} \int_{0}^{L} x C_{1 k}$ $(x, t) \mathrm{d} x \mathrm{~d} t-\Theta \int_{0}^{L} x f_{C k}(x) \mathrm{d} x+\Theta \frac{L^{2}}{2}, \gamma_{k}=3$

$\alpha_{2 c k}=\sqrt[3]{\sqrt{q^{2}+p^{3}}-q}-\sqrt[3]{\sqrt{q^{2}+p^{3}}+q}-\frac{a_{10}}{a_{9}}$

where $\quad q=\frac{a_{10}^{3}}{27 a_{9}^{3}}-\frac{a_{10} a_{11}}{3 a_{9}^{2}}+\frac{a_{12}}{a_{9}}, p=\frac{a_{11}}{3 a_{9}}-\frac{a_{10}^{2}}{9 a_{9}^{2}}, a_{9}=\xi_{k} \int_{0}^{\Theta}$ $(\Theta-t) \int_{0}^{L} R_{03 k}(x, t) \mathrm{d} x \mathrm{~d} t, a_{10}=\xi_{k} \int_{0}^{\Theta}(\Theta-t) \times \int_{0}^{L} R_{13 k}(x$, $t) \mathrm{d} x \mathrm{~d} t, a_{9}=\xi_{k} \int_{0}^{\Theta}(\Theta-t) \int_{0}^{L} R_{23 k}(x, t) \mathrm{d} x \mathrm{~d} t+\Theta \frac{L^{2}}{2}, a_{12}=$ $\int_{0}^{\Theta} \int_{0}^{L} x C_{1 k}(x, t) \mathrm{d} x \mathrm{~d} t-\int_{0}^{L} f_{C k}(x) \times x \mathrm{~d} x \Theta+\xi_{k} \int_{0}^{\Theta}(\Theta-t)$ $\int_{0}^{L} R_{33 k}(x, t) \mathrm{d} x \mathrm{~d} t+\int_{0}^{\Theta}(\Theta-t) \int_{0}^{L} R_{00 k}(x, t) \mathrm{d} x \mathrm{~d} t$.

\section{References}

Fahey PM, Griffin PB, Plummer JD (1989) Point defects and dopant diffusion in silicon. Rev Mod Phys 61:289

Gotra ZYu (1991) Technology of microelectronic devices (Radio and communication, Moscow, in Russian)

Grebene AB (1983) Bipolar and MOS analogous integrated circuit design. Wiley, New York

Gusev VG, Gusev YuM (1991) Electronics (High school, Moscow, in Russian)

Lachin VI, Savelov NS (2001) Electronics (Phoenix, Rostov-naDonu, in Russian)

Pankratov EL (2007) Dynamics of delta-dopant redistribution during heterostructure growth. Eur Phys J B 57(3):251

Pankratov EL (2008a) Redistribution of dopant, implanted in a multilayer structure for production of a p-n-junction, during annealing radiation defects. Phys Lett A 372(11):1897

Pankratov EL (2008b) Redistribution of dopant during microwave annealing of a multilayer structure for production $\mathrm{p}-\mathrm{n}$-junction. J Appl Phys 103(6):064320 
Pankratov EL (2008c) Analysis of redistribution of radiation defects with account diffusion and several secondary processes. Mod Phys Lett B 22(28):27779

Sokolov YuD (1955) About determination dynamical stress in hoisting ropes of mines. Appl Mech 1(1):23
Zorin EI, Pavlov PV, Tetelbaum DI (1975) Ion doping of semiconductors. Energiya, Moscow (in Russian) 